\title{
CARACTERÍSTICAS CLÍNICO EPIDEMIOLÓGICAS DE LOS CASOS DE TUBERCULOSIS INFANTIL DIAGNOSTICADOS EN EL HOSPITAL INFANTIL DR. ROBERT REID CABRAL 2007- 2012
}

\section{Clinical epidemiological characteristics of cases of childhood Tuberculosis diagnosed in the Dr. Robert Reid Cabral Children's Hospital 2007-2012}

\author{
Ricardo Elías Melgen*, Elsa Camilo Pantaleón**, Adria Lazala J***, Aimee Abreu B***
}

Fecha de recibido: octubre 4, 2018 • Fecha de aprobado: diciembre 12, 2018

Cómo citar: Melgen R E, Pantaleón E C, Lazala J A, Abreu B. A. Características clínico epidemiológicas de los casos de tuberculosis infantil diagnosticados en el Hospital Infantil Dr. Robert Reid Cabral 2007- 2012. cysa [Internet]. 10 abr. 2019 [citado 12 abr. 2019];1(1):9-3. Disponible en: https://revistas.intec.edu.do/index.php/cisa/article/ view/1325

\section{Resumen}

Introducción: la TB infantil es considerada como un evento centinela de transmisión de la enfermedad y su control no ha sido una prioridad. República Dominicana es un país de elevada incidencia de TB y los datos reportados de TB infantil apuntan a una baja detección de casos y un sub diagnóstico.

Objetivo: conocer las características clínico epidemiológicas de los casos de Tuberculosis infantil diagnosticados en el Hospital Infantil Dr. Robert Reid Cabral. 2007- 2012.

Métodos: estudio descriptivo. Se revisaron los expedientes clínicos de los pacientes menores de 15 años de edad diagnosticados y seguidos en el programa de tuberculosis infantil del Hospital Infantil Robert Reid Cabral en los años 2007-2012

Resultados: se evaluaron 249 pacientes, $81 \%$ con diagnóstico de TB infantil y 19\% con Infección Latente por Tuberculosis. $56.2 \%$ tenía menos de 5 años de edad y

\footnotetext{
* Departamento de Neumología, Hospital Infantil Dr. Robert Reid Cabral, Centro Nacional de Investigación en Salud Materno Infantil Dr. Hugo Mendoza (CENISMI).

reliasmelgen@yahoo.es

** Departamento de Neumología, Hospital Infantil Dr. Robert Reid Cabral. elsacamilo@hotmail.com
}

61.4\% tenían cicatriz de vacuna BCG. $63.5 \%$ de los casos refería el contacto con un caso de Tuberculosis, y en el $70.0 \%$ era intradomiciliario. La manifestación pulmonar representó el $63.4 \%$ de los casos TB y el $27.3 \%$ tenían baciloscopia positiva.

Conclusión: existen diferencias importantes en el diagnóstico y manejo de la tuberculosis infantil y la TB en el adultos. Estas deben ser considerados en la implementación de estrategias para el control; con acciones integradas, un enfoque orientado no solo al niño, sino también a la familia y tomando en consideración los determinantes sociales.

Palabras clave: Tuberculosis; Tuberculosis infantil; Coinfección TB/SIDA; Diagnóstico Tuberculosis infantil; Epidemiología Tuberculosis infantil

\section{Abstract}

Background: Childhood TB is considered a sentinel event of transmission of the disease and its control has not been a priority. The Dominican Republic is a country with a high incidence of TB and the reported data on childhood $\mathrm{TB}$ point to a low detection of cases and a sub-diagnosis

\footnotetext{
***. Residencia Neumología Pediátrica, Hospital Infantil Dr. Robert Reid Cabral. dralazalaj@hotmail.com

Hospital Infantil Robert Reid Cabral, República Dominicana. Email: aimee_abreu@hotmail.com
} 
Objective: To know the clinical epidemiological characteristics of cases of childhood Tuberculosis diagnosed in the Dr. Robert Reid Cabral Children's Hospital 2007- 2012.

Methods: descriptive study. We reviewed the clinical records of patients under 15 years of age diagnosed and followed in the childhood tuberculosis program of the Robert Reid Cabral Children's Hospital in the years 2007-2012

Results: 249 patients were evaluated, $81 \%$ with a diagnosis of childhood TB and 19\% Latent Tuberculosis Infection. $56.2 \%$ had less than 5 years of age and $61.4 \%$ had BCG vaccine scar. $63.5 \%$ of the cases referred to the contact with a case of Tuberculosis, and in $70.0 \%$ it was intradomiciliary. Pulmonary manifestation represented $63.4 \%$ of TB cases and $27.3 \%$ had smear positive.

Conclusion: there are important differences in the diagnosis and management of childhood tuberculosis and TB in adults, these should be considered in the implementation of strategies for control; with integrated actions, an approach oriented not only to the child, but also to the family and taking into consideration the social determinants.

Keywords: tuberculosis; Childhood Tuberculosis; TB / AIDS co-infection; Diagnosis Tuberculosis in children; Epidemiology Tuberculosis in children

\section{Introducción}

Se define Tuberculosis Infantil a todo paciente menor de 15 años de edad, que se diagnostica Tuberculosis (TB) y se decide iniciar tratamiento antituberculosis, ${ }^{1}$ representa un indicador de infección reciente, principalmente en menores de 5 años de edad, sugiriendo la existencia de casos bacilífero no diagnosticado.

La TB infantil es considerada como un evento centinela de transmisión de la enfermedad. ${ }^{2}$ y que por su condición paucibacilar tiene poco impacto epidemiológico; pero que desde el punto de vista clínico representa un reto para establecer su diagnóstico. ${ }^{3}$

República Dominicana es un país de elevada incidencia por TB y los datos reportados de TB infantil apunta a una baja detección de casos y un sub diagnóstico. ${ }^{4,5}$ Tradicionalmente el control de la Tuberculosis infantil no ha sido una prioridad, pero al ser esta una población vulnerable se debe conocer su comportamiento y así poder establecer estrategias para su control. ${ }^{6}$

Diferentes factores favorecen a que un niño se infecte y desarrolle la enfermedad por tuberculosis, entre éstos: la edad, antecedentes de contacto con un caso enfermo, hacinamiento, infección con el $\mathrm{VIH}$, desnutrición y la no vacunación con BCG. ${ }^{3}$

El objetivo de la presente investigación es conocer las características clínico epidemiológicas de los casos de Tuberculosis infantil diagnosticados en el Hospital Infantil Dr. Robert Reid Cabral 2007- 2012.

\section{Material y método}

Estudio descriptivo. Se revisaron los expedientes clínicos de los pacientes menores de 15 años de edad diagnosticados y seguidos en el programa de tuberculosis infantil del Hospital Infantil Robert Reid Cabral en los años 2007-2012.

Se excluyeron aquellos pacientes diagnosticados fuera del período de estudio, paciente mayor de la edad establecida, casos con Tuberculosis multidrogoresistente.

Los datos fueron recolectados utilizando un formulario previamente diseńado y validado para tales fines, organizados y tabulados, mediante IBM SPSS Statistisc 22.

Toda información recolectada se utilizó bajo estricta confidencialidad por parte de los investigadores, y no se dieron a conocer datos pertinentes de los pacientes y sus familiares.

\section{Resultados}

Durante el periodo de estudio se evaluaron 249 pacientes de los cuales 202 (81\%) fueron 
diagnosticados como TB infantil y 47 (19\%) como Infección Latente por Tuberculosis.

No hubo diferencia en cuanto al sexo, 50.2\% (125) masculino; el 56.2\% (140) tenía menos de 5 años de edad y el $61.4 \%$ (153) presentaban cicatriz de vacuna $B C G$.

El 71.5\% (178) del total de los casos evaluados residían en Santo Domingo, $18.9 \%$ (47) vivían en hacinamiento, más de 3 personas por habitación y en el $63.5 \%$ (158) de los casos de Tuberculosis se refería haber estado en contacto con un caso de Tuberculosis, siendo este contacto intradomiciliario en el $70.0 \%$ (109).

En el $30.9 \%$ de los casos el/la jefe/a de familia era la madre y el 9.2\% vivía con los abuelos. En el 57\% de los casos el/la jefe/a de familia apenas alcanzaba el nivel primario de escolaridad, de estos el 7.2\% no había asistido a la escuela y en el $48.6 \%$ era desempleado/a. (Tabla 1)

Tabla 1. Características Socio demográficos

\begin{tabular}{lcc}
\hline & $\begin{array}{c}\text { Frecuencia } \\
(\mathrm{N}=249)\end{array}$ & $\mathbf{\%}$ \\
\hline Jefe/a de familia & 146 & 58.6 \\
Padre & 77 & 30.9 \\
Madre & 23 & 9.2 \\
Abuelos & 3 & 1.2 \\
Otros & & \\
Escolaridad & & \\
jefe/a de familia & 124 & 49.8 \\
Primaria & 84 & 33.7 \\
Secundaria & 21 & 8.4 \\
Universitaria & 2 & 0.8 \\
Técnico & 18 & 7.2 \\
Ninguna & & \\
Ocupación & & \\
jefe/a de familia & 128 & 51.4 \\
Empleado & 121 & 48.6 \\
Desempleado & & \\
\hline
\end{tabular}

Fuente: Expedientes clínicos Departamento Neumología HIRRC.
En el 68.7\% (171) se refería algún antecedente familiar patológico: Tuberculosis $47.0 \%$, VIH/SIDA 6.8\%, Asma 7.6\%, Diabetes 6.0\%, Hipertensión arterial $2.8 \%$.

En el 66.3\% (165) de los casos se refería algún antecedente personal patológico: Tuberculosis $2.4 \%$, VIH/SIDA 6.0\%, Asma y sibilancia recurrente $34.5 \%$, Desnutrición $14.8 \%$. (Tabla 2)

Tabla 2. Antecedentes familiares y personales

\begin{tabular}{lcc}
\hline & $\begin{array}{c}\text { Frecuencia } \\
(\mathrm{N}=249)\end{array}$ & \% \\
\hline Familiares & 117 & 47.0 \\
Tuberculosis & 17 & 6.8 \\
VIH/SIDA & 15 & 6.0 \\
Diabetes & 19 & 7.6 \\
Asma & 7 & 2.8 \\
Hipertensión & & \\
Personales & 6 & 2.4 \\
Tuberculosis & 15 & 6.0 \\
VIH/SIDA & & \\
Asma y sibilancia & 86 & 34.5 \\
recurrente & 37 & 14.8 \\
Desnutrición & & \\
\hline
\end{tabular}

Fuente: Expedientes clínicos Departamento Neumología HIRRC.

Con relación a la sintomatología, la fiebre (41.8\%), tos $(36.5 \%)$ y adenopatías (25.7\%) fueron las manifestaciones clínicas más frecuente.

En los casos de TB diagnosticados, la manifestación pulmonar representó el 63.4\% (128/202) y el $27.3 \%$ (35) presentó baciloscopia positiva.

La manifestación extrapulmonar se diagnóstico en el 36.6\% (74/202) de los casos, siendo la ganglionar con el $78.4 \%$ la más frecuente, seguida por la meníngea con $13.5 \%$ (ver tabla 3 ). 
Tabla 3. Manifestaciones de los casos con TB Extrapulmonar

\begin{tabular}{lcc}
\hline Manifestaciones EP & Frecuencia & \% \\
\hline Ganglionar & 58 & 78.4 \\
Meníngea & 10 & 13.5 \\
Columna & 3 & 4.1 \\
Abdominal & 1 & 1.4 \\
Miliar & 2 & 2.7 \\
Total & $\mathbf{7 4}$ & $\mathbf{1 0 0 . 0}$ \\
\hline
\end{tabular}

Fuente: Expedientes clínicos Departamento Neumología HIRRC.

No se pudo establecer el resultado al tratamiento en el 13.6\% (34) de los casos, 85.9\% (214) completaron exitosamente el esquema de tratamiento recomendado y un caso de TB fracasó al tratamiento antituberculosis.

\section{Discusión}

La tuberculosis sigue siendo una causa importante y poco reconocida de enfermedad y muerte en los niños que viven en zonas de elevada incidencia; su magnitud se desconoce, pero la Organización Mundial de la Salud (OMS), estima que la tuberculosis infantil representa aproximadamente un $10 \%$ de todos los nuevos casos de TB.

La dificultad en poder establecer un diagnóstico definitivo en los niños por su condición paucibacilar y el hecho de que como los niños no hacen una contribución significativa a la propagación de la enfermedad, tradicionalmente su control no ha sido una prioridad para los programas de control de TB en los países. ${ }^{7}$

El porcentaje de casos de tuberculosis en menores de 15 ańos está relacionado con la incidencia de la enfermedad, la pirámide poblacional y el nivel de desarrollo socioeconómico de una población. ${ }^{8} \mathrm{La}$ transmisión y progresión a enfermedad está relacionada a factores sociales y económicos; mejorar las condiciones en los niveles de vida y tener un enfoque social de la enfermedad, ayudó a disminuir la carga de la TB antes de la introducción de la quimioterapia en la década de $1940 .{ }^{9}$

Es evidente, como la población estudiada vivía en pobres condiciones social y económica; $18.9 \%$ de los casos evaluados vivía en hacinamiento, el responsable familiar no alcanzaba el nivel primario de escolaridad en el $57 \%$ de los casos y de estos el 7.2\% no había asistido a la escuela; el responsable familiar no tenía empleo en el $48.6 \%$ y en el $30.9 \%$ de los casos la madre era la responsable familiar.

La tuberculosis infantil es un indicador de transmisión reciente y continua del bacilo de la TB en la comunidad. El contacto cercano con un caso de TB pulmonar, especialmente aquellos con bacteriología positiva, es el principal factor de riesgo para la TB en los niños; ${ }^{3}$ en el $63.5 \%$ de los casos evaluados se refirió el haber estado en contacto con un caso de TB, siendo este contacto intradomiciliario en el $70.0 \%$.

Los niños pueden enfermar por tuberculosis a cualquier edad, pero en los países con elevada carga, el rango más frecuente es entre 1 y 4 años, ${ }^{6}$ el 56.2\% de los casos evaluados tenía menos de 5 años de edad. Igualmente, la infección por el VIH y la desnutrición son factores de riesgo importantes en el desarrollo de la tuberculosis; el $6 \%$ de los casos presentaba VIH/SIDA y el 14.8\% tenían algún grado de desnutrición.

Desde el punto de vista clínico, la TB infantil representa un reto por la dificultad de confirmar el diagnóstico y por la similitud de las manifestaciones clínicas con otras enfermedades de la infancia. Una evaluación exhaustiva de todas las pruebas derivadas a partir de una historia cuidadosa de la exposición, el examen clínico y las investigaciones pertinentes, son fundamentales. ${ }^{6}$

La manifestación pulmonar es el tipo más frecuente de tuberculosis en los niños, la extrapulmonar 
puede representar alrededor del 30-40 \% de los casos, presentándose en una amplia variedad de sitios anatómicos. ${ }^{6} \mathrm{El} 36.6 \%$ de los casos evaluados presentaron manifestación extrapulmonar; localizándose en ganglio (78.4\%), meninges $(13.5 \%)$, columna $(4.1 \%)$, miliar $(2.7 \%)$ y abdominal $(1.4 \%)$.

El hecho de que la tuberculosis pulmonar en niños generalmente es paucibacilar, esto no debe impedir el uso de baciloscopia y cultivo como uno de los criterios principales para establecer el diagnóstico. El 27.3\% de los casos con manifestación pulmonar evaluados, presentaron baciloscopia positiva.

\section{Conclusión}

Los niños representan una población particularmente vulnerable, existen diferencias importantes en el diagnóstico y manejo de la tuberculosis infantil y la TB en adultos, y éstas deben ser consideradas en la implementación de estrategias para el control.

Se deben tomar acciones integradas con un enfoque orientado no solo al niño, sino también a la familia; facilitando el acceso universal a diagnóstico y un tratamiento de TB de alta calidad, tomando en consideración los determinantes sociales que son en gran medida los responsables de la endemia.

\section{Referencias}

1. Ministerio de Salud Pública. "Reglamento técnico para la prevención y el control de la Tuberculosis” MSP/VGC/RT02/14. República Dominicana, 2014.
2. Lancella, L. How to manage children who have come in to contact with patients affected by tuberculosis. Journal of Clinical Tuberculosis and Other Mycobacterial Diseases 1 (2015) $1-12$

3. Farga, V; Caminero, J.A. "Tuberculosis" La UNION. 3ra. Edición. 2011.

4. Ministerio de Salud Pública. "Sistema de Información Operacional y Epidemiológica del Programa Nacional de Control de la Tuberculosis”. República Dominicana. 2014.

5. World Health Organization. "Global Tuberculosis Report 2014"

6. World Health Organization. Guidance for national tuberculosis programmes on the management of tuberculosis in children. Second edition. 2014

7. Parés-Badell, O., Volz G, A., Victoria, J., del Granado C M., Situación de la Tuberculosis en la población infantil en la Región de Las Américas, 2013 Neumol Pediatr 2015; 10 (4): $148-154$

8. Donald PR. Childhood tuberculosis: out of control? Curr Opin Pulm Med 2002

9. Ortblad KF, Salomon JA, Barnighausen T, Atun R., Stopping tuberculosis: a biosocial model for sustainable developmentwww.thelancet.com Published online October 26, 2015 http:// dx.doi.org/10.1016/S0140-6736(15)00324-4 